\title{
Increasing market efficiency in the stock markets
}

Jae-Suk Yang ${ }^{1}$, Wooseop Kwak ${ }^{1, a}$, Taisei Kaizoji ${ }^{2}$, and In-mook Kim¹,3,b

1 Department of Physics, Korea University, Seoul 136-701, Korea

2 Division of Social Sciences, International Christian University, Osawa, Mitaka, Tokyo 181-8585, Japan

3 School of Physics, Korea Institute for Advanced Study, Seoul 130-722, Korea

Eur. Phys. J. B 61, 241 (2008)

Received 20 February 2008

Published online 29 February 2008 - (c) EDP Sciences, Società Italiana di Fisica, Springer-Verlag 2008

The following sentence was missing in the article [Jae-Suk Yang, Wooseop Kwak, Taisei Kaizoji, In-mook Kim, Eur. Phys. J. B 61, 241 (2008)]:

"This work is supported in part by the Second Brain Korea 21 project and also by the Korea University Grant."

\footnotetext{
${ }^{a}$ Current address: Department of Physics, Chosun University, Gwangju, 501-759, Korea.

b e-mail: imkim@korea.ac.kr
} 